\title{
Approche couplée éléments discrets/finis pour la simulation d'un impact sur ouvrage
}

\author{
Emmanuel Frangin — Philippe Marin — Laurent Daudeville \\ Laboratoire 3S-R (sols, solides, structures, risques) \\ Université Joseph Fourier - CNRS - Institut National Polytechnique de Grenoble \\ Domaine Universitaire - BP 53, F-38041 Grenoble cedex 9 \\ \{frangin, marin2, daudeville\}@hmg.inpg.fr
}

\begin{abstract}
RÉSUMÉ. Pour étudier la réponse d'un ouvrage à des sollicitations dynamiques sévères de type impact, nous utilisons une modélisation mixte permettant de tirer profit des avantages de la méthode des éléments discrets dans les zones proches de l'impact et susceptibles de s'endommager fortement. Le reste de la structure est modélisé par la méthode des éléments finis. Cette approche permet de modéliser l'ensemble de l'ouvrage avec une discrétisation et un coût de calcul raisonnables et permet également de modéliser la multifracturation de la cible et l'éventuelle pénétration du projectile. Ce papier discute des moyens d'atténuer les réflexions d'ondes parasites dues au changement de taille de la discrétisation et présente une application sur une dalle en béton impactée par un bloc rocheux, application permettant de comparer la réponse de la structure lors de la modélisation mixte avec celle utilisant uniquement des éléments discrets.

ABSTRACT. The paper deals with combined finite/discrete element method to study structures under severe dynamic loading, like impact. The discrete element method take naturally into account the non linear phenomena, it is used in the vicinity of the impacted zone. The finite element method is used to reduce the time of computation in order to carry out large structure analyses. The aim of the paper is to discuss the way to reduce non physical wave reflections; it presents an application on a rock impact on a concrete slab.

MOTS-CLÉS : éléments discrets, dynamique, propagation d'onde, impact, ondes parasites.

KEYWORDS: discrete element, dynamics, wave propagation, impact, spurious waves.
\end{abstract}

DOI:10.3166/REMN.16.989-1009 @ 2007 Lavoisier, Paris 


\section{Introduction}

Certains ouvrages nécessitent une attention particulière lors de leur dimensionnement pour évaluer leur comportement sous sollicitations dynamiques sévères. D'origine naturelle ou anthropique, un ou des impacts sur des structures en béton armé peuvent avoir des conséquences dramatiques, par exemple si l'évènement conduit à une perforation d'un ouvrage devant assurer une fonction de protection ou d'étanchéité. Ce papier propose une nouvelle méthode couplant différentes approches en fonction de la réponse de la structure. Elle repose sur une partition de la structure en deux domaines. Le premier domaine, proche de l'impact, est le lieu d'importants endommagements, de nombreux phénomènes non-linéaires (fissurations, fragmentation, écaillage). Le second domaine concerne le reste de la structure qui est supposé subir de petites perturbations. Le domaine fortement endommagé est modélisé par la méthode des éléments discrets, tandis que la partie élastique de la structure est modélisée par la méthode des éléments finis. Les expériences d'impact sur des structures en béton (Berriaud et al., 1978) ou l'utilisation des règles de conception, Eurocode 2, permettent d'avoir une estimation de la taille de la zone fortement endommagée en fonction des caractéristiques dimensionnelles ou énergétiques de l'impact.

Contrairement à une approche continue classique, la méthode des éléments discrets, composée d'éléments rigides en interaction, représente naturellement les phénomènes discontinus et elle est utilisée dans la zone impactée. Ces approches ont été développées pour les matériaux granulaires (Cundall et al., 1979), mais sont également bien adaptées pour des géomatériaux cohésifs comme le béton (D'addetta et al., 2002). Les travaux de Camborde en 2D (Camborde et al., 2000a) ou Hentz en 3D (Hentz et al., 2005) ont montré la pertinence de l'utilisation des éléments discrets (ED) pour les problèmes d'impact. Ils ont également montré leur limitation quant à la taille des structures modélisables (Hentz, 2003). L'utilisation d'éléments finis (EF) loin de la zone impactée permet de lever cette limitation en réduisant les temps de modélisation et de simulation (Frangin et al., 2006).

De nombreux travaux sont en cours concernant les approches couplées entre différents modèles. Ben Dhia (BenDhia, 1998) propose un outil de couplage (méthode Arlequin) utilisant une formulation faible pour prendre en compte les conditions de couplage sur une zone de recouvrement entre deux domaines de discrétisation et/ou de modélisation différente pour des applications en statiques. D'autres travaux sont également menés sur le couplage entre des éléments finis et des méthodes sans maillage. Une revue de ces approches est proposée dans ( $\mathrm{Li}$ et al., 2004). Appliquées aux structures en béton, différentes méthodes ont été proposées, en statique (Azevedo et al., 2006; Cusatis et al., 2006). En dynamique, (Oñate et al., 2004) traitent le contact entre EF et ED, (Bicanic et al., 1997) proposent une méthode transformant les EF qui deviennent discrets en fonction d'un critère. Les premiers couplages modélisant une partie du domaine par ED et une autre par EF ont été effectués par les physiciens à l'échelle atomique (Broughton et al., 1999; Rudd et al., 1998). Les travaux de (Xiao et al., 2004) préconisent un couplage avec recouvrement comme Ben Dhia, mais ils utilisent une formulation discrète des conditions de couplage, formulation mieux adaptée 
aux éléments discrets pour lesquels les champs de déplacement et de déformation ne sont pas facilement explicites. Ils proposent par ailleurs un traitement numérique réduisant les réflexions d'ondes parasites au niveau de la zone de transition. Par rapport à cette dernière approche, le travail que nous présentons doit prendre en considération la partie rotation des ED que nous utilisons. De plus, nos simulations montrent que les simplifications proposées par Belytschko n'atténuent pas suffisamment les ondes réfléchies et nous proposons une variante pour la prise en compte temporelle des conditions aux limites pour améliorer l'atténuation des ondes réfléchies.

Après avoir présenté les principales caractéristiques des approches discrètes et continues, nous détaillons l'approche couplée et les différentes difficultés à relever, ainsi que les équations permettant de résoudre le problème. L'étude de la propagation des ondes nous permet de définir une solution pour réduire les ondes parasites. L'utilisation d'un amortissement visqueux n'a pas été retenue en raison des difficultés de choix de la valeur de cet amortissement et de son influence néfaste sur le pas de temps critique de notre schéma d'intégration explicite. Enfin nous démontrons la faisabilité et l'efficacité de la méthode par la simulation de l'impact d'un bloc rocheux sur une dalle en béton. Cet exemple nous permet de comparer la solution issue d'une méthode mixte avec celle obtenue avec uniquement des éléments discrets et illustre les gains de temps potentiels.

\section{Description de l'approche}

Le problème à résoudre est un problème de dynamique transitoire dans lequel la structure est divisée en deux sous-domaines. Un domaine est modélisé par la méthode des éléments discrets, tandis que l'autre est modélisé par la méthode des éléments finis. La séparation des deux domaines est réalisée à partir de la taille du domaine endommagé et de celle du domaine supposé sous l'hypothèse des petites perturbations. Cette étape est, dans notre cas, réalisée a priori en fonction des connaissances empiriques (Berriaud et al., 1978) de la zone endommagée, elle-même fonction de la taille et de la vitesse de l'impacteur. Les règles de construction de l'Eurocode 2 préconisent la taille des zones fortement sollicitées. Une méthode adaptative transformant les EF en ED au cours de la simulation lorsque les éléments discrets proches de la zone de recouvrement s'endommagent, pourrait être implantée, mais elle n'est pas l'objet de cette étude.

\subsection{Modèle discret}

Le domaine où apparaissent les discontinuités et les non-linéarités, est modélisé par la méthode des ED. De nombreuses simulations ont montré les performances de ces méthodes pour analyser la réponse des structures en béton sous fortes déformations et non-linéarités importantes que ce soit en 2D, (Camborde et al., 2000b), ou en 3D (Hentz et al., 2004a), en statique (Cusatis et al., 2006) ou en dynamique (Donzé et al., 1999). Le modèle utilisé est celui proposé par (Cundall et al., 1979). On s'in- 
téresse à un assemblage d'éléments sphériques désordonné, afin de représenter le caractère hétérogène et isotrope du béton. Ces éléments sphériques sont reliés entre eux par des lois d'interaction non-linéaires, de type contact ou lien cohésif. Afin d'obtenir le comportement cohésif du matériau, deux éléments qui ne sont pas en contact, peuvent être en interaction de type lien. La description du modèle discret utilisé est présentée dans les travaux de (Hentz et al., 2004b). Notons toutefois que les ED possèdent trois degrés de liberté supplémentaires aux EF, les trois rotations de l'espace. Le modèle discret que nous représentons ne respecte pas la taille des granulats. Nous nous situons à une échelle mésoscopique. La puissance de calcul disponible ne nous permet pas de descendre à une échelle inférieure et cela ne représente pas un objectif en soi. Toutefois, afin d'améliorer la prédiction de l'endommagement et de respecter la physique, la taille des éléments sera aussi fine que possible, tout en conservant un rapport entre les quelques gros éléments et les nombreux petits éléments de l'ordre de 10. Enfin, un processus d'identification des propriétés matériaux a été développé au cours de travaux antérieurs (Hentz et al., 2004b), il permet d'obtenir un modèle prédictif de la réponse des structures.

\subsection{Modèle continu}

Loin de la zone d'impact, la structure est modélisée par la méthode des EF sous l'hypothèse des petites perturbations. Considérer que la structure (suffisamment loin de la zone impactée) reste linéaire élastique peut paraître une hypothèse forte $a$ priori, mais rien n'empêche a postériori de déterminer les zones endommagées et de les modéliser dans une seconde simulation avec une zone discrète plus importante. Il suffit pour cela de vérifier que les ED proches des EF n'ont pas été endommagés. Nous nous intéressons dans ce papier au problème de couplage entre les deux méthodes. Pour cela, dans l'exemple traité, les deux zones sont séparées et l'endommagement est très localisé. Le choix du couplage avec les EF a pour but de diminuer le temps de modélisation des structures de forme complexe, par l'utilisation de logiciel de maillage, mais également le temps de simulation en diminuant très fortement le nombre d'ED. La taille des EF est fonction de la géométrie de la structure, elle est largement supérieure à la taille des ED (rapport de taille supérieur à 5).

\subsection{Difficultés de couplage}

La méthode proposée utilise une zone de recouvrement. Il y a deux raisons principales à cela : d'une part, la répartition désordonnée des ED conduirait à la réalisation d'un maillage particulier pour coupler bord à bord les éléments. D'autre part, un traitement adapté de la zone de recouvrement permet dans certains cas de réduire les ondes parasites apparaissant dans la zone de recouvrement (section 4). Par ailleurs, le modèle ED a trois degrés de liberté de plus par nœud que le modèle EF. Si les déplacements ED sont naturellement liés aux déplacements EF, il est également nécessaire de coupler les rotations ED aux ddl EF. Dans l'hypothèse des petites perturbations, l'ex- 
périence (Calvetti et al., 1997) et les simulations montrent que les rotations des ED correspondent au vecteur rotation des EF, égal à la partie antisymétrique du gradient de la transformation. Dans le modèle continu et en HPP, le déplacement d'un point $\mathrm{N}$ dans le voisinage de M s'exprime par :

$$
\vec{U}(N) \cong \vec{U}(M)+\overline{\bar{\epsilon}}(M) \overrightarrow{M N}+\vec{\Omega}(M) \wedge \overrightarrow{M N}
$$

Le déplacement s'écrit comme la somme de la translation de corps rigide, de la partie due à la déformation et à la rotation.

Autre difficulté, la taille de discrétisation n'est pas la même entre les deux approches. La densité du maillage ou la densité des ED définissent la gamme de fréquence que peut représenter chaque modèle. Le passage de la discrétisation fine à la discrétisation grossière d'un signal contenant une large gamme de fréquence entraîne la réflexion des ondes hautes fréquences au niveau de l'interface. La zone de recouvrement est vue comme un mur pour les fréquences supérieures à la fréquence de coupure des EF.

La figure 1 montre un exemple de cette réflexion d'ondes parasites par l'interface sur un exemple 1D. Il s'agit d'une barre modélisée sur la moitié droite par un maillage ED régulier fin et sur la partie gauche par un maillage EF régulier grossier. Une sollicitation en déplacement combinant basses et hautes fréquences est imposée sur le bord droit. Les basses fréquences sont transmises, tandis que les hautes fréquences sont réfléchies au niveau de l'interface. La même réponse est obtenue en utilisant uniquement des ED ou des EF. L'utilisation d'un maillage EF progressif entraine les mêmes réflexions. Afin de ne pas modifier la physique du modèle dans la zone discrète, il est nécessaire de développer une méthode qui supprime ces ondes parasites. Pour des essais expérimentaux d'impacts à vitesse moyenne $(10 \mathrm{~m} / \mathrm{s})$, les réponses mesurées contiennent des hautes fréquences de quelques kilohertz (Zineddin et al., 2007), supérieures à la fréquence de coupure de EF. Il est donc nécessaire de traiter avec rigueur ce problème de réflexion.

\section{Méthode de couplage avec recouvrement}

\subsection{Notations}

$\begin{array}{ll}\vec{d} & \text { vecteur des déplacements des ED } \\ \vec{\omega} & \text { vecteur des rotations des ED } \\ \vec{u} & \text { vecteur des déplacements nodaux des EF } \\ \overrightarrow{d r} & \text { vecteur des déplacements des ED de la zone de recouvrement } \\ \overrightarrow{\omega r} & \text { vecteur des rotations des ED de la zone de recouvrement } \\ \overrightarrow{u r} & \text { vecteur des déplacements nodaux des EF de la zone de recouvrement } \\ \overrightarrow{d_{j}} & \text { vecteur des trois composantes du déplacement de l'ED } j\end{array}$




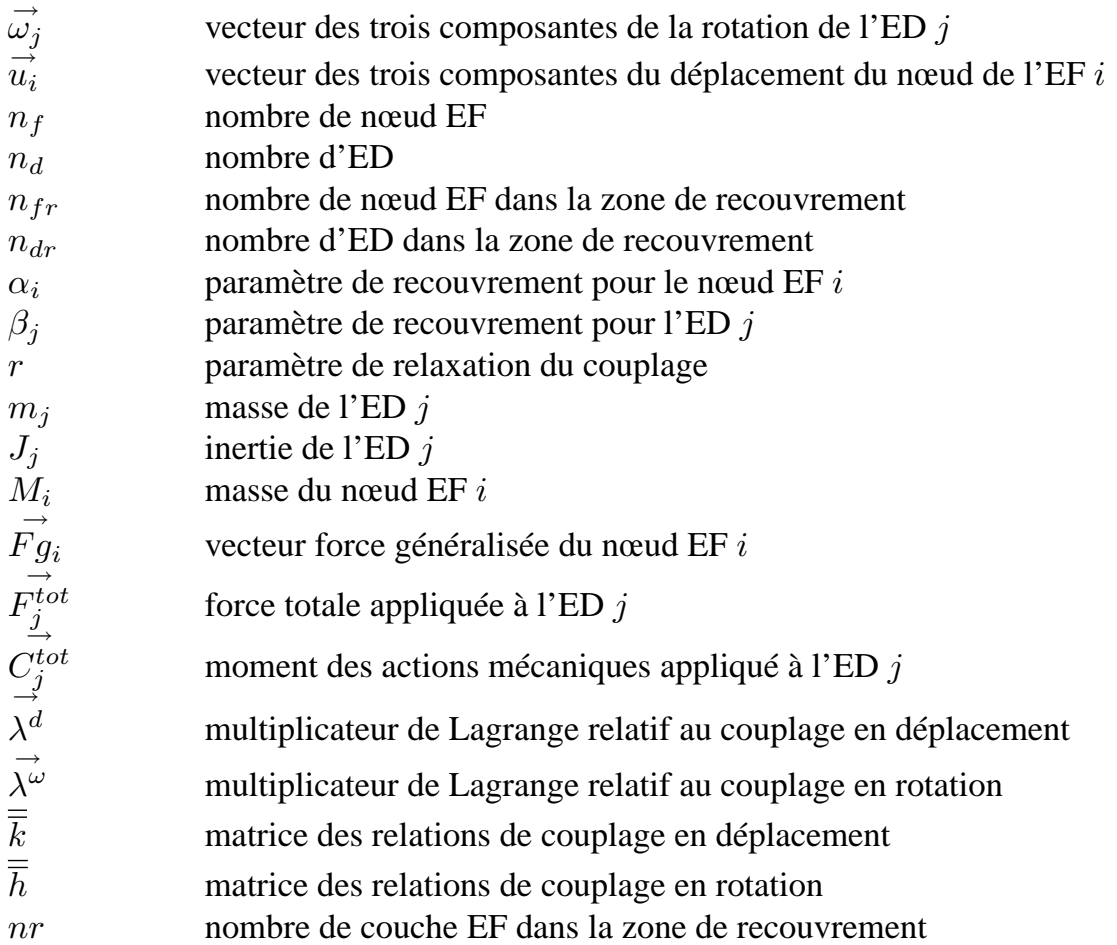

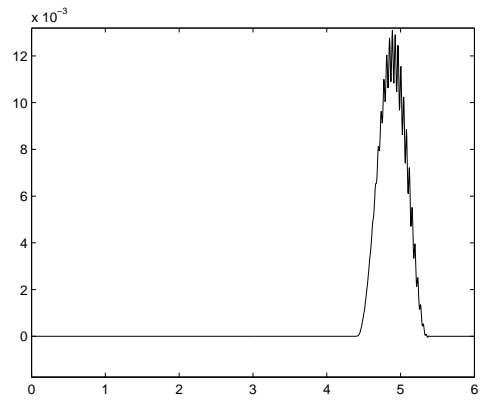

(a) Signal dans la partie discrète

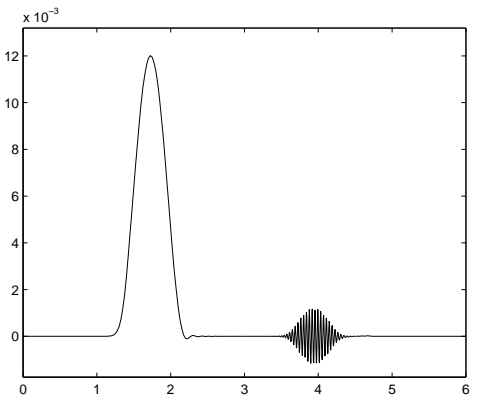

(b) Réflexion de la haute fréquence

Figure 1. Réflexions des ondes hautes fréquences sur l'interface entre une discrétisation fine et grossière 


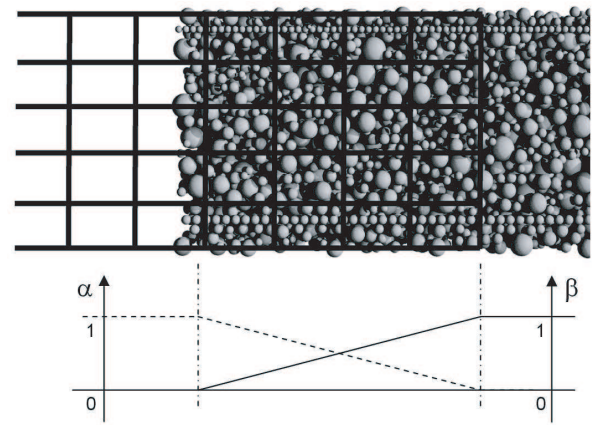

Figure 2. Zone de recouvrement et paramètres de recouvrement

\subsection{Méthodologie}

La méthode par recouvrement utilise dans la zone de superposition, un Hamiltonien, combinaison linéaire du Hamiltonien discret et du Hamiltonien continu. Les paramètres de recouvrement, $\alpha$ et $\beta$, sont introduits respectivement sur les nœuds EF et les ED. Ils varient linéairement entre 0 et 1 le long du recouvrement et ils sont constants dans l'épaisseur. Ces paramètres sont équivalents à ceux utilisés par Xiao (Xiao et al., 2004). Ils permettent d'assurer de façon continue la partition énergétique entre les domaines modélisés par EF et par ED. On peut parler de zone de transition. Dans la méthode Arlequin (BenDhia, 1998), le paramètre de recouvrement utilisé n'assure pas nécessairement ce passage progressif entre les domaines, ce qui peut introduire des réflexions supplémentaires.

La taille de la zone de recouvrement est définie par le paramètre $n_{r}$, le nombre de couche d'EF à utiliser. Pour un maillage donné, plus $n_{r}$ est grand plus la zone de recouvrement est grande. La figure 2 montre la variation des paramètres de recouvrement sur un exemple où $n_{r}=4$. L'équation [2] donne l'expression du Hamiltonien.

$$
H=\alpha \cdot H_{E F}+\beta \cdot H_{E D}
$$

Dans la zone de recouvrement, les ddl des ED sont liés aux ddl des EF par les relations cinématiques de couplage, définies par les équations [3] et [4], ou au niveau des nœuds des ED par [5] et [6].

$$
\begin{aligned}
& \overrightarrow{d r}=\overline{\bar{k}} \cdot \overrightarrow{u r} \\
& \overrightarrow{\omega r}=\overline{\bar{h}} \cdot \overrightarrow{u r}
\end{aligned}
$$




$$
\begin{aligned}
& \forall j \leq n_{d r} \quad \overrightarrow{d_{j}}=\sum_{i=1}^{n_{f r}} \overline{\overline{k_{j i}}} \overrightarrow{u_{i}} \\
& \forall j \leq n_{d r} \quad \overrightarrow{\omega_{j}}=\sum_{i=1}^{n_{f r}} \overline{\overline{h_{j i}}} \overrightarrow{u_{i}}
\end{aligned}
$$

Les matrices $\overline{\bar{k}}$ et $\overline{\bar{h}}$ sont structurées en sous-matrices $\overline{\overline{k_{j i}}}, \overline{\overline{h_{j i}}}$ relatives au nœud EF $i$ et ED $j$ (figure 3).

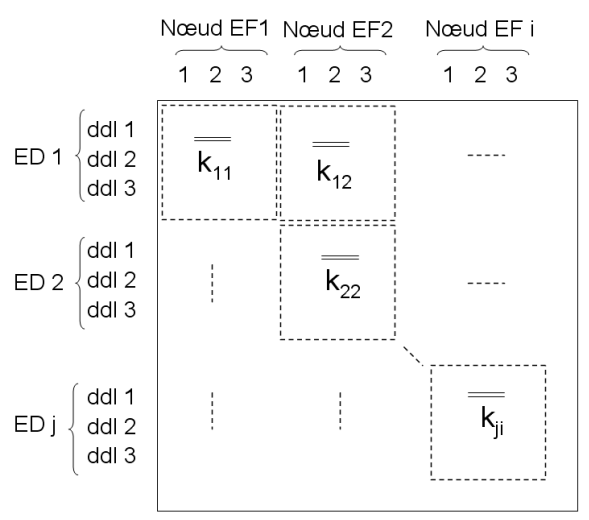

Figure 3. Organisation des matrices de couplage en sous-matrices

La solution minimise le Hamiltonien sur chaque domaine avec introduction des relations de couplage dans la zone de recouvrement par multiplicateurs de Lagrange. $\overrightarrow{\lambda^{d}}$ est le multiplicateur de Lagrange relatif au couplage des déplacements. $\overrightarrow{\lambda^{\omega}}$ est le multiplicateur de Lagrange relatif au couplage des rotations. La solution est obtenue en minimisant l'expression [7], elle conduit aux équations locales.

$$
\begin{array}{r}
H g\left(\vec{d}, \vec{\omega}, \vec{u}, \overrightarrow{\lambda^{d}}, \overrightarrow{\lambda^{\omega}}\right)=H(\vec{d}, \vec{\omega}, \vec{u})+ \\
\overrightarrow{\lambda^{d}}(\overrightarrow{d r}-\overline{\bar{k}} \overrightarrow{u r})+\overrightarrow{\lambda^{\omega}}(\overrightarrow{\omega r}-\overline{\bar{h}} \overrightarrow{u r})
\end{array}
$$

- Equation locale d'un nœud ED $j$ :

$$
\begin{aligned}
& m_{j} \overrightarrow{\ddot{d}_{j}}=\overrightarrow{F_{j}^{t o t}} \\
& J_{j} \overrightarrow{\ddot{\omega}_{j}}=C_{j}^{t o t}
\end{aligned}
$$


- Equation locale d'un nœud EF $i$ (Nous utilisons une matrice masse EF condensée aux nœuds) :

$$
M_{i} \overrightarrow{\ddot{u}_{i}}=\overrightarrow{F g_{i}}
$$

- Dans la zone de recouvrement :

$$
\begin{aligned}
& \forall j \leq n_{d r} \quad \beta_{j} m_{j} \quad \overrightarrow{\ddot{d}_{j}}=\beta_{j} \overrightarrow{F_{j}^{t o t}}+\overrightarrow{\lambda_{j}^{d}} \\
& \forall j \leq n_{d r} \quad \beta_{j} J_{j} \quad \overrightarrow{\ddot{\omega}_{j}}=\beta_{j} \overrightarrow{C_{j}^{t o t}}+\overrightarrow{\lambda_{j}^{\omega}} \\
& \forall i \leq n_{f r} \quad \alpha_{i} M_{i} \quad \overrightarrow{\ddot{u}}_{i}=\alpha_{i} \overrightarrow{F g_{i}}+\sum_{l=1}^{n_{d r}} \overrightarrow{\vec{k}_{l i}} T \overrightarrow{\lambda_{l}^{d}}+\sum_{l=1}^{n_{d r}}{\overrightarrow{\overline{h_{l i}}}}^{T} \overrightarrow{\lambda_{l}^{\omega}}
\end{aligned}
$$

\subsection{Algorithme}

La discrétisation en temps est réalisée par la méthode des différences finies centrées.

$$
\begin{aligned}
& U(t+\Delta t)=U(t)+\Delta t \dot{U}\left(t+\frac{\Delta t}{2}\right) \\
& \dot{U}\left(t+\frac{\Delta t}{2}\right)=\dot{U}\left(t-\frac{\Delta t}{2}\right)+\Delta t \ddot{U}(t) \\
& U(t+\Delta t)=2 U(t)-U(t-\Delta t)+\Delta t^{2} \ddot{U}
\end{aligned}
$$

Les ddl au pas de temps $n+1$ s'expriment donc par :

$$
\begin{aligned}
& \vec{d}_{j}(t+\Delta t)=2 \vec{d}_{j}(t)-\vec{d}_{j}(t-\Delta t)+\frac{\Delta t^{2}}{m_{j}}\left(\overrightarrow{F_{j}^{t o t}}+\frac{\overrightarrow{\lambda_{j}^{d}}}{\beta_{j}}\right) \\
& \overrightarrow{\omega_{j}}(t+\Delta t)=2 \overrightarrow{\omega_{j}}(t)-\vec{\omega}_{j}(t-\Delta t)+\frac{\Delta t^{2}}{J_{j}}\left(\overrightarrow{C_{j}^{t o t}}+\frac{\overrightarrow{\lambda_{j}^{\omega}}}{\beta_{j}}\right) \\
& \vec{u}_{i}(t+\Delta t)=2 \vec{u}_{i}(t)-\vec{u}_{i}(t-\Delta t)+\frac{\Delta t^{2}}{M_{i}} \overrightarrow{F g}_{i}+ \\
& \frac{\Delta t^{2}}{\alpha_{i} M_{i}}\left(\sum_{l=1}^{n_{d r}}{\overline{\overline{k_{l i}}}}^{T} \overrightarrow{\lambda_{l}^{d}}+\sum_{l=1}^{n_{d r}}{\overline{\overline{h_{l i}}}}^{T} \overrightarrow{\lambda_{l}^{\omega}}\right)
\end{aligned}
$$


Dans un premier temps, une valeur provisoire des ddl à $t+\Delta t$ est déterminée sans prendre en considération les multiplicateurs de Lagrange :

$$
\begin{gathered}
\overrightarrow{\tilde{d}_{j}}(t+\Delta t)=2 \overrightarrow{d_{j}}(t)-\overrightarrow{d_{j}}(t-\Delta t)+\frac{\Delta t^{2}}{m_{j}} \overrightarrow{F_{j}^{t o t}} \\
\overrightarrow{\tilde{\omega}_{j}}(t+\Delta t)=2 \overrightarrow{\omega_{j}}(t)-\vec{\omega}_{j}(t-\Delta t)+\frac{\Delta t^{2}}{J_{j}} \overrightarrow{C_{j}^{t o t}} \\
\overrightarrow{\tilde{u}_{i}}(t+\Delta t)=2 \overrightarrow{u_{i}}(t)-\vec{u}_{i}(t-\Delta t)+\frac{\Delta t^{2}}{M_{i}} \overrightarrow{F g_{i}}
\end{gathered}
$$

Les $\overrightarrow{u_{i}}, \overrightarrow{d_{j}}, \overrightarrow{\omega_{j}}$ à $t+\Delta t$ doivent vérifier les équations de couplage. Cela impose aux multiplicateurs $\overrightarrow{\lambda_{j}^{d}}$ et $\overrightarrow{\lambda_{l}^{\omega}}$ de vérifier les relations suivantes :

$$
\begin{aligned}
& \overrightarrow{g_{j}^{d}}=\overrightarrow{\tilde{d}_{j}}(t+\Delta t)-\sum_{i=1}^{n_{f r}} \overline{\overline{k_{j i}}} \overrightarrow{\tilde{u}_{i}}(t+\Delta t) \\
& =\Delta t^{2}\left(-\frac{\overrightarrow{\lambda_{j}^{d}}}{\beta_{j} m_{j}}-\sum_{i=1}^{n_{f r}} \overline{\overline{k_{j i}}}\left(\sum_{l=1}^{n_{d r}} \frac{{\overline{\overline{k_{l i}}}}^{T} \overrightarrow{\lambda_{l}^{d}}+{\overline{\overline{h_{l i}}}}^{T} \overrightarrow{\lambda_{l}^{\omega}}}{\alpha_{i} M_{i}}\right)\right) \\
& \overrightarrow{g_{j}^{\omega}}=\overrightarrow{\tilde{\omega}_{j}}(t+\Delta t)-\sum_{i=1}^{n_{f r}} \overline{\overline{h_{j i}}} \overrightarrow{\tilde{u}_{i}}(t+\Delta t) \\
& =\Delta t^{2}\left(-\frac{\overrightarrow{\lambda_{j}^{\omega}}}{\beta_{j} J_{j}}-\sum_{i=1}^{n_{f r}} \overline{\overline{h_{j i}}}\left(\sum_{l=1}^{n_{d r}} \frac{{\overrightarrow{\overline{k_{l i}}}}^{T}{\overrightarrow{\lambda_{l}^{d}}}^{d}+{\overline{\overline{h_{l i}}}}^{T} \overrightarrow{\lambda_{l}^{\omega}}}{\alpha_{i} M_{i}}\right)\right)
\end{aligned}
$$

où $\overrightarrow{g_{j}^{d}}$ et $\overrightarrow{g_{j}^{\omega}}$ sont des résidus intermédiaires des relations des couplages, avant la prise en compte des multiplicateurs de Lagrange.

Ces expressions s'écrivent sous forme matricielle :

$$
\begin{aligned}
& \overrightarrow{g^{d}}=\overline{\bar{A}} \overrightarrow{\lambda^{d}}+\overline{\bar{B}} \overrightarrow{\lambda^{\omega}} \\
& \overrightarrow{g^{\omega}}=\overline{\bar{C}} \overrightarrow{\lambda^{d}}+\overline{\bar{D}} \overrightarrow{\lambda^{\omega}}
\end{aligned}
$$

où

$$
\overline{\overline{A_{j l}}}=-\frac{\Delta t^{2}}{\beta_{j} m j} \overline{\overline{I d_{33}}}-\Delta t^{2} \sum_{p=1}^{n_{f r}} \frac{\overline{\overline{k_{j p}}}{\overline{\overline{k_{l p}}}}^{T}}{\alpha_{p} M_{p}}
$$




$$
\begin{aligned}
& \overline{\overline{B_{j l}}}=-\Delta t^{2} \sum_{p=1}^{n_{f r}} \frac{\overline{\overline{k_{j p}}}{\overline{\overline{h_{l p}}}}^{T}}{\alpha_{p} M_{p}} \\
& \overline{\overline{C_{j l}}}=-\Delta t^{2} \sum_{p=1}^{n_{f r}} \frac{\overline{\overline{h_{j p}}} \overline{\overline{k_{l p}}}}{\bar{\alpha}_{p} M_{p}} \\
& \overline{\overline{D_{j l}}}=-\frac{\Delta t^{2}}{\beta_{j} J_{j}} \delta_{j l}-\Delta t^{2} \sum_{p=1}^{n_{f r}} \frac{\overline{\bar{h}_{j p}}}{{\overline{h_{l p}}}^{\alpha_{p} M_{p}}}
\end{aligned}
$$

La résolution de ce système matriciel permet de calculer les multiplicateurs de Lagrange et ensuite les déplacements et les rotations à $t+\Delta t$ en utilisant les relations [17], [18], [19].

\subsection{Simplifications du schéma numérique}

L'analyse des différentes matrices montre la prédominance de $\overline{\bar{A}}$ et $\overline{\bar{D}}$ devant les matrices $\overline{\bar{B}}$ et $\overline{\bar{C}}$, les masses ED étant beaucoup plus faibles que les masses des EF. Si l'on néglige les deux matrices $\overline{\bar{B}}$ et $\overline{\bar{C}}$, le schéma numérique obtenu est équivalent à une méthode particulière de pénalité (cf. 3.5). Les multiplicateurs sont alors approximés par :

$$
\begin{aligned}
& \overrightarrow{\lambda^{d}}=\overline{\bar{A}}^{-1} \overrightarrow{g^{d}} \\
& \overrightarrow{\lambda^{\omega}}=\overline{\bar{D}}^{-1} \overrightarrow{g^{\omega}}
\end{aligned}
$$

Par ailleurs, les termes diagonaux des matrices étant prédominants sur les autres valeurs, nous utilisons la simplification proposée par (Xiao et al., 2004), simplification que nous utilisons également pour la prise en compte des rotations des ED. Les matrices $\overline{\bar{A}}$ et $\overline{\bar{D}}$ sont remplacées par des matrices diagonales, dont le terme diagonal est obtenu en sommant les termes de la ligne correspondante. Cette simplification procure deux avantages, un gain de temps par l'utilisation des matrices diagonales et également une légère amélioration des résultats en termes d'atténuation des ondes réfléchies parasites. Toutefois, les simulations de la propagation d'onde (section 4) montrent que cette atténuation est insuffisante et nous proposons par la suite l'ajout d'un paramètre de relaxation de l'influence des multiplicateurs de Lagrange pour augmenter cette atténuation. Nous démontrons ci-après que les simplifications présentées reviennent à utiliser une méthode particulière de pénalité au lieu de la méthode par multiplicateur de Lagrange proposée, en ayant l'avantage d'obtenir une valeur du coefficient de pénalité automatiquement et d'une façon adaptée à chaque ddl. 


\subsection{Equivalence avec la méthode de pénalité}

L'équivalence de la méthode proposée utilisant la diagonalisation des matrices des multiplicateurs $\overline{\bar{A}}$ et $\overline{\bar{D}}$ et négligeant les matrices $\overline{\bar{B}}$ et $\overline{\bar{C}}$ avec une variante de la méthode de pénalité est démontrée sur l'équation locale des déplacements d'un nœud ED. La même démonstration peut être effectuée facilement sur les autres ddl.

En utilisant la relation [31, la mise à jour des ddl est obtenue avec les équations suivantes :

$$
\overrightarrow{d_{j}}(t+\Delta t)=2 \overrightarrow{d_{j}}(t)-\vec{d}_{j}(t-\Delta t)+\frac{\Delta t^{2}}{m_{j}}\left(\overrightarrow{F_{j}^{t o t}}+\frac{{\overline{A_{j j}}}^{-1} \overrightarrow{g_{j}^{d}}}{\beta_{j}}\right)
$$

La méthode de pénalité sous une forme générale revient à minimiser :

$$
\begin{aligned}
& H(\vec{d}, \vec{\omega}, \vec{u})+(\overrightarrow{d r}-\overline{\bar{k}} \overrightarrow{u r})^{T} \overline{\overline{p^{d}}}(\overrightarrow{d r}-\overline{\bar{k}} \overrightarrow{u r})+ \\
& (\overrightarrow{\omega r}-\overline{\bar{h}} \overrightarrow{u r})^{T} \overline{\overline{p^{\omega}}}(\overrightarrow{\omega r}-\overline{\bar{h}} \overrightarrow{u r})
\end{aligned}
$$

où $\overline{\overline{p^{d}}}$ et $\overline{\overline{p^{\omega}}}$ sont dans le cas général deux matrices symétriques définies positives. Elles sont prises ici comme c'est souvent le cas diagonales. Les équations locales obtenues avec la méthode par pénalité s'écrivent pour le déplacement d'un nœud ED par :

$$
\beta_{j} m_{j} \overrightarrow{d_{j}}=\beta_{j} \overrightarrow{F_{j}^{t o t}}+\overline{\overline{p_{j j}^{d}}}\left(\overrightarrow{d_{j}}-\sum_{i=1}^{n_{f r}} \overline{\overline{k_{j i}}} \overrightarrow{u_{i}}\right)
$$

En utilisant le schéma aux différences centrées, on obtient :

$$
\begin{aligned}
\vec{d}_{j}(t+\Delta t)= & 2 \overrightarrow{d_{j}}(t)-\overrightarrow{d_{j}}(t-\Delta t)+ \\
& \frac{\Delta t^{2}}{m_{j}}\left(\overrightarrow{F_{j}^{t o t}}+\frac{\overline{\overline{p_{j j}^{d}}}\left(\overrightarrow{d_{j}}-\sum_{i=1}^{n_{f r}} \overline{\overline{k_{j i}}} \overrightarrow{u_{i}}\right)}{\beta_{j}}\right)
\end{aligned}
$$

Or $\left(\overrightarrow{d r}-\sum_{i=1}^{n_{f r}} \overline{\overline{k_{j i}}} \overrightarrow{u_{i}}\right)$ est égal à $\overrightarrow{g_{j}^{d}}$ avec nos notations. Les équations [33] et [36] sont donc équivalentes si l'on prend ${\overline{\overline{A_{j j}}}}^{-1}=\overline{\overline{p_{j j}^{d}}}$. La généralisation à l'ensemble des ddl ne présente aucune difficulté. Avec cette approche, chaque équation de couplage a son propre paramètre de pénalité, calculé automatiquement. 


\section{Analyse de la propagation des ondes}

\subsection{Réflexion des hautes fréquences}

Pour tout type de simulation en régime transitoire, la taille de la discrétisation introduit une fréquence de coupure propre au modèle. En effet, pour qu'une onde se propage sans être modifiée, il faut avoir environ un rapport supérieur à 10 entre sa longueur d'onde et la taille des éléments. Or, dans notre modèle, les deux parties sont discrétisées à deux échelles différentes, finement pour les ED afin d'améliorer la prédiction de l'endommagement et plus grossièrement en EF pour diminuer les temps de simulation. Une onde haute fréquence supérieure à la fréquence de coupure de la partie EF sera alors réfléchie au niveau de la zone de recouvrement ; cela induit une onde réfléchie sans signification physique que l'on doit faire disparaître ou à défaut atténuer.

\subsection{Méthode d'atténuation : la relaxation}

Avec la méthode lagrangienne proposée, les conditions de couplage sur les ddl sont rigoureusement respectées, c'est-à-dire que les ddl des ED sont complètement définis à partir des ddl des éléments finis, et donc dépendent de la discrétisation grossière. Ce couplage conduit à une réflexion complète des ondes hautes fréquences. L'utilisation de matrices diagonales, présentée en 3.4, a pour intérêt de réduire le temps de simulation. Cette simplification (Xiao et al., 2004) réduit également très légèrement les ondes réfléchies (figure 4), mais cette réduction est insuffisante. Afin d'atténuer fortement les ondes parasites, nous proposons de relâcher les conditions cinématiques en limitant l'effet des multiplicateurs de Lagrange par l'introduction d'un paramètre de relaxation, $r$. La correction utilisée lors de la mise à jour des ddl n'est plus proportionnelle à $\lambda$, mais à $\frac{\lambda}{r}$.

$$
\begin{aligned}
& \vec{u}_{i}(t+\Delta t)= 2 \vec{u}_{i}(t)-\vec{u}_{i}(t-\Delta t)+ \\
& \frac{\Delta t^{2}}{M_{i}}\left(\overrightarrow{F g_{i}}+\sum_{l=1}^{n_{d r}} \frac{{\overline{k_{l i}}}^{T} \overrightarrow{\lambda_{l}^{d}}+{\overline{\bar{h}_{l i}}}^{T} \overrightarrow{\lambda_{l}^{\omega}}}{\alpha_{i} r}\right) \\
& \vec{d}_{j}(t+\Delta t)=2{\overrightarrow{d_{j}}}_{(t)-\vec{d}_{j}}(t-\Delta t)+\frac{\Delta t^{2}}{m_{j}}\left(\overrightarrow{F_{j}^{t o t}}+\frac{\overrightarrow{\lambda_{j}^{d}}}{\beta_{j} r}\right)
\end{aligned}
$$

En ajoutant ainsi un peu de liberté sur les relations de couplage, les ondes réfléchies sont atténuées. Les figures 45 et 6 montrent de manière quantitative pour un 
problème 1D l'influence de ces différentes méthodes sur le pourcentage d'atténuation de l'énergie contenue dans l'onde réfléchie. Avec l'algorithme lagrangien rigoureux, l'énergie contenue dans la haute fréquence est complètement réfléchie. Avec une méthode combinant la diagonalisation de la matrice des multiplicateurs de Lagrange et en utilisant un paramètre de relaxation, cette énergie réfléchie est quasiment annulée.

$$
\overrightarrow{\omega_{j}}(t+\Delta t)=2 \overrightarrow{\omega_{j}}(t)-\overrightarrow{\omega_{j}}(t-\Delta t)+\frac{\Delta t^{2}}{J_{j}}\left(\overrightarrow{C_{j}^{t o t}}+\frac{\overrightarrow{\lambda_{j}^{\omega}}}{\beta_{j} r}\right)
$$

La figure 4 est établie en utilisant une matrice diagonale non relaxée. L'atténuation de l'énergie réfléchie provient alors uniquement de l'écriture sous forme diagonale des matrices. Cette simplification permet d'atténuer de près de $40 \%$ l'énergie réfléchie par l'interface. Cette atténuation varie légèrement en fonction de la taille de la zone de recouvrement ( $n_{r}=0$ correspond à un raccordement surfacique).

Les figures 5 et 6 montrent l'influence du paramètre de relaxation pour différentes tailles de zone de recouvrement. La première est obtenue en considérant les matrices des multiplicateurs complètes, la seconde est obtenue avec des matrices diagonalisées. L'utilisation de deux couches de recouvrement permet d'atténuer de plus de 80 $\%$ l'énergie contenue dans l'onde réfléchie par l'interface. La figure 7 compare la propagation des ondes entre l'approche avec multiplicateur de Lagrange rigoureuse (trait plein) et la méthode utilisant une matrice diagonale et un paramètre de relaxation égal au nombre de couche d'ED dans la zone de recouvrement (pointillé). Dans cet exemple, la taille de la zone de recouvrement est égale à trois couches EF. On peut noter que l'onde transmise est quasi identique (variations négligeables) et que l'onde réfléchie est fortement atténuée.

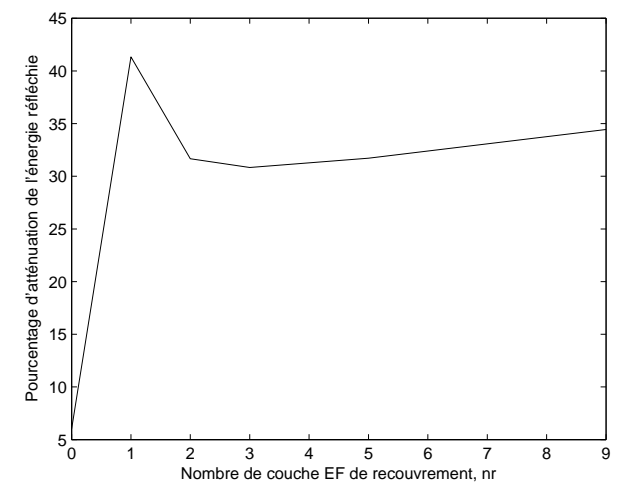

Figure 4. Influence de la diagonalisation en fonction de la taille de la zone de recouvrement sur le pourcentage d'atténuation de l'énergie réfléchie 


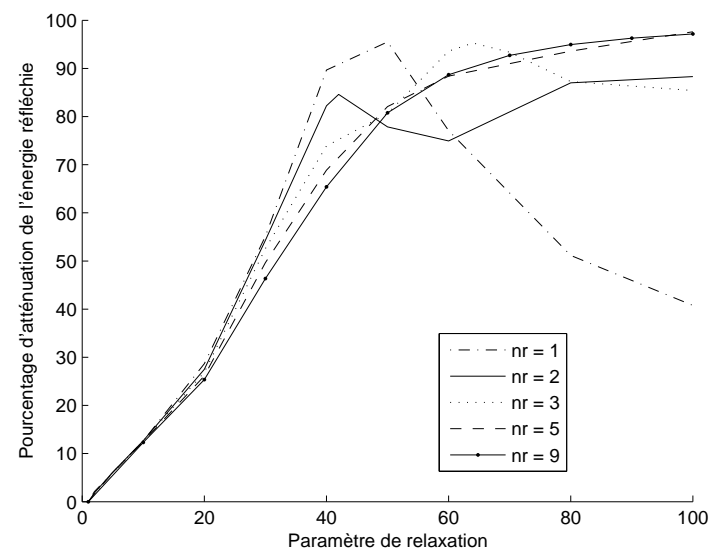

Figure 5. Influence du paramètre de relaxation (sans diagonalisation des matrices) sur le pourcentage d'atténuation de l'énergie réfléchie pour différentes tailles de la zone de recouvrement

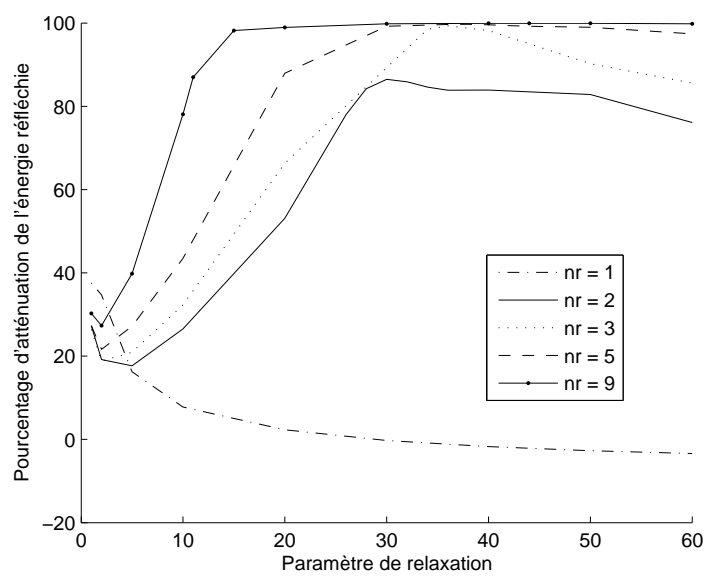

Figure 6. Influence du paramètre de relaxation (avec des matrices diagonales) sur le pourcentage d'atténuation de l'énergie réfléchie pour différentes tailles de la zone de recouvrement 


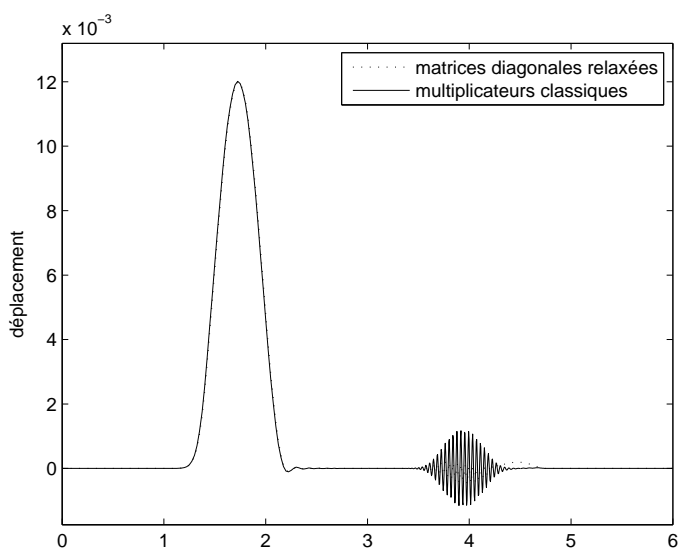

(a) Ondes transmises et réfléchies

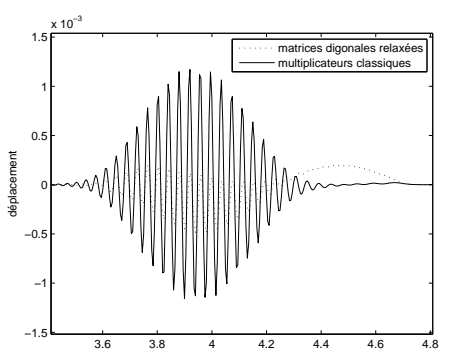

(b) Onde HF réfléchie

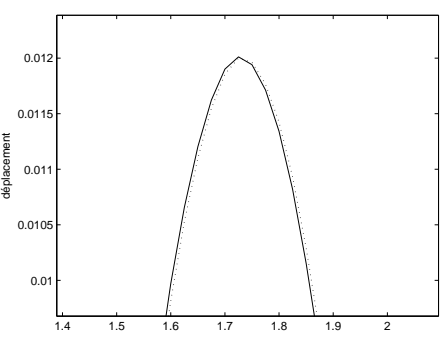

(c) Onde BF transmise

Figure 7. Etude de la propagation des ondes selon la méthode, multiplicateurs classiques (plein), matrices diagonales relaxées (pointillé)

\section{Simulation 3D d'un impact sur une dalle en béton}

Dans cette section, nous souhaitons comparer les résultats obtenus pour une formulation discrète avec ceux obtenus pour une formulation mixte (ED/EF) en termes de précision et de temps de calcul. L'exemple traité est l'impact d'un bloc rocheux sur une dalle en béton. Les expériences réalisées par (Zineddin et al., 2007) sur des dalles en béton armé montrent la nécessité de prendre en considération dans la partie endommagement la présence des phénomènes à faible longueur d'onde. La réflexion des hautes fréquences dans la zone de recouvrement de la méthode mixte est prise en compte par l'utilisation de la méthode présentée précédemment. Dans cette simulation, nous comparons les résultats en déplacement, en endommagement, ainsi que le coût en temps de calcul pour le modèle aux éléments discrets uniquement et le modèle 
couplé ED/EF utilisant trois couches de recouvrement (figure 8) et un paramètre de relaxation égal à 40. Le rapport de discrétisation ED/EF est égal à cinq.

La figure 8 définit la géométrie du modèle. La taille de la dalle est de 2,5 x 2 × 0,3m. Le modèle EF est constitué d'éléments hexaédriques avec cinq couches d'éléments dans l'épaisseur pour représenter le comportement en flexion de la dalle.

La figure 9 présente la comparaison en déplacement entre les deux modèles. Les deux premières figures comparent les déplacements ED dans la zone discrète, la suivante ceux dans la zone de recouvrement et la dernière compare le déplacement d'un nœud EF à celui de l'ED le plus proche.

Pour comparer l'endommagement de la structure, on se place à deux instants de temps différents, $T 1=2,5 \cdot 10^{-5} \mathrm{sec}$ et $T 2=1 \cdot 10^{-4} \mathrm{sec}$. L'endommagement est défini comme le rapport entre le nombre de liens cassés sur le nombre de liens initiaux (figure 10). Plus l'élément est sombre, plus il est endommagé.

Les résultats en déplacement et en endommagement sont très satisfaisants. Les courbes sont comparables en amplitude.

Ce couplage vise à diminuer le temps de calcul pour être en mesure d'étudier une structure complète. Le tableau 1 résume les caractéristiques du problème couplé et du problème ED. Dans cet exemple, l'approche couplée est dix fois plus rapide que l'approche purement discrète. Ce facteur dépend fortement de la taille de la zone ED. En négligeant le temps de calcul pour les éléments finis, le facteur de réduction du temps de calcul est directement fonction du rapport entre le nombre d'ED du problème purement discret et celui du problème mixte. Par ailleurs, l'algorithme présenté peut facilement utiliser des pas de temps différents pour le modèle ED et pour le modèle $\mathrm{EF}$, ce qui rend assez négligeable le temps consacré aux EF. En réduisant ainsi le temps de calcul, on peut envisager des structures plus grandes ou des discrétisations plus raffinées.

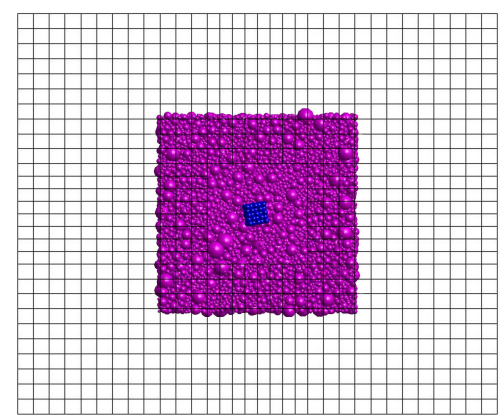

Figure 8. Description du modèle avec les domaines EF, ED, la zone de recouvrement et l'impacteur 

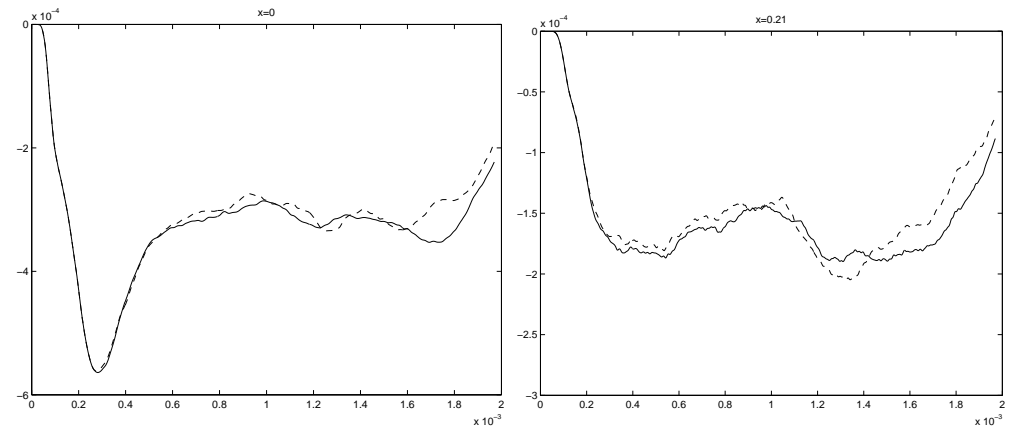

(a) Déplacement d'ED sous l'impac- (b) Déplacement d'un ED à $0.21 \mathrm{~m}$ de teur

l'impact
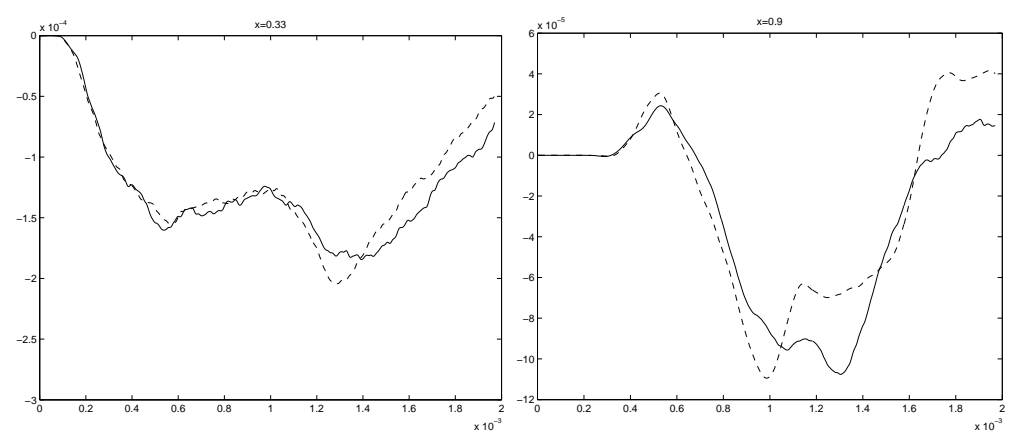

(c) Déplacement d'un ED à $0.33 \mathrm{~m}$ de (d) Comparaison du déplacement d'un l'impact nœud EF et d'un ED

Figure 9. Comparaison des déplacements entre le modèle discret (trait plein) et le modèle couplé avec 3 couches de recouvrement (courbe pointillée)

\begin{tabular}{|c|c|c|}
\hline Modèle & ED seul & Approche couplée \\
\hline Nombre d'ED & 120808 & 6588 \\
\hline Nombre de nœud EF & $\mathrm{X}$ & 5935 \\
\hline $\begin{array}{c}\text { Nombre de couche EF } \\
\text { dans la zone de recouvrement }(\mathrm{nr}=3)\end{array}$ & $\mathrm{X}$ & 3 \\
\hline Nombre de pas de temps & 100000 & 100000 \\
\hline Temps de simulation & $42 \mathrm{~h} 50 \mathrm{mn}$ & $3 \mathrm{~h} 39 \mathrm{mn}$ \\
\hline
\end{tabular}

Tableau 1. Comparaison entre ED et ED/EF 


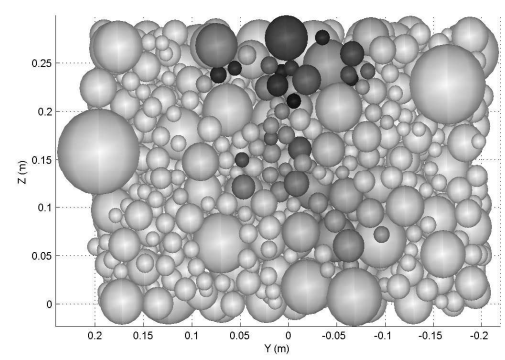

(a) Modèle discret au temps 1

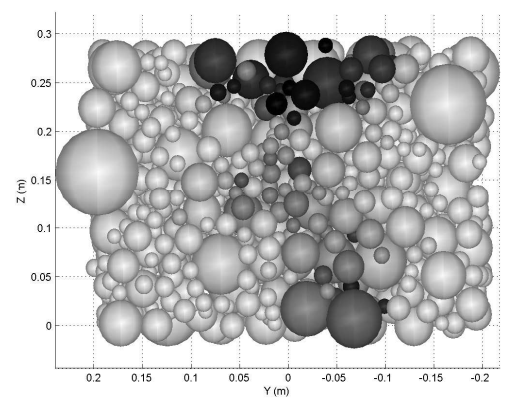

(c) Modèle discret au temps 2

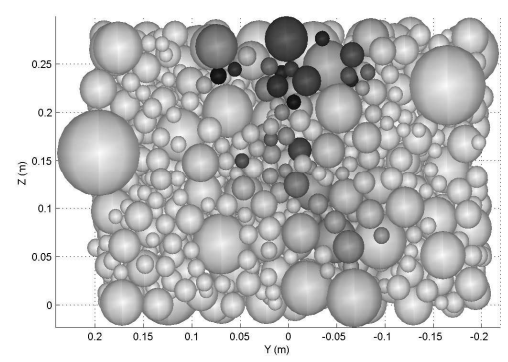

(b) Modèle mixte au temps 1

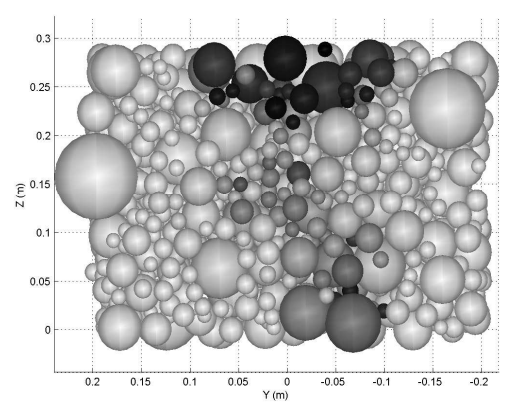

(d) Modèle mixte au temps 2

Figure 10. Comparaison de l'endommagement entre le modèle discret (a), (c) et le modèle couplé avec 3 couches de recouvrement $(b),(d)$

\section{Conclusion}

La méthode couplée proposée permet de traiter les problèmes d'impact en divisant la structure en deux parties en fonction du comportement de chacune d'entre-elle. Le couplage entre les deux approches se fonde sur une approche avec des multiplicateurs de Lagrange et un traitement numérique particulier pour réduire les réflexions des ondes parasites. Celles-ci apparaissent du fait de la variation de la taille de la discrétisation. En plus de la diagonalisation des matrices liées aux multiplicateurs, nous proposons d'atténuer l'effet des multiplicateurs par l'introduction d'un paramètre de relaxation sur la prise en compte de ces multiplicateurs. Cela permet d'atténuer en quasi-totalité les ondes réfléchies. L'algorithme ainsi transformé est équivalent à une méthode de pénalité.

Pour la simulation d'un ouvrage, le modèle couplé conserve le caractère prédictif de la méthode discrète et également la qualité des résultats locaux, déplacements et endommagement des ED. Il permet également de décrire le comportement de l'ouvrage dans son ensemble. Le gain de temps obtenu par cette approche, nous permet d'envisager d'étudier des structures de plus grande taille avec une discrétisation locale plus 
fine qu'avec un modèle constitué uniquement d'éléments discrets. Les applications $3 \mathrm{D}$ réalisées en utilisant la méthode diagonale relaxée nous ont montré son aptitude à représenter le comportement des ouvrages sous impacts.

\section{Bibliographie}

Azevedo N., Lemos J., « Hybrid discrete element/finite element method for fracture analysis », Comput. Methods Appl. Mech. Engrg., 2006.

BenDhia H., «Problèmes mécaniques multi-échelle : la méthode Arlequin », C. R. Acad. Sci. Paris, vol. 326, p. 899-904, 1998.

Berriaud C., Sokolovsky A., Gueraud R., Dulac J., Labrot R., « Comportement local des enceintes en béton sous l'impact d'un projectile rigide », Nuclear Engineering and Design, vol. 45, p. 457-469, 1978.

Bicanic N., Munjiza A., Owen D. R. J., Petrinic N., « From Continua to Discontinua - a Combined Finite Element/Discrete Element Modelling in Civil Engineering », Dynamic Modelling of Geomaterials, ALERT geomaterials, 1997.

Broughton J. Q., Abraham F. F., Bernstein N., Kaxiras E., « Concurrent coupling of length scales : Methodology and application », Physical review B, July, 1999.

Calvetti F., Combe G., Lanier J., « Experimental Micromechanical Analysis of a 2D Granular Material : Relation Between Structure Evolution and Loading Path», Mechanics of cohesive-frictional Materials, vol. 2, $\mathrm{n}^{\circ}$ 2, p. 121-163, 1997.

Camborde F., Grillon Y., Chaigneau F., « Discrete Element Method for Predicting the Behaviour of Concrete under Dynamic Loading », Journal de physique IV (Proceedings), vol. 10, n ${ }^{\circ}$, p. 467-74, 2000a.

Camborde F., Mariotti F. C., Donzé F., « Numerical Study of Rock and Concrete Behaviour by Discrete Element Modelling », Computers and geotechnics, vol. 27, n 4, p. 225-247, 2000 b.

CEB, Concrete structure under impact and impulsive loading, Comité Euro-international du béton, 1988.

Cundall P. A., Strack O. D. L., « A Discrete Numerical Model for Granular Assemblies », Geotechnique, vol. 29, n 1, p. 47-65, 1979.

Cusatis G., Bazant Z. P., Cedolin L., « Confinement-shear lattice CSL model for fracture propagation in concrete», Comput.Methods Appl. Mech. Engrg., 2006.

D'addetta G. A., Kun F., Ramm E., « On the application of a discrete model to the fracture process of cohesive granular materials », Granular Matter, vol. 4, p. 77-90, 2002.

Donzé F. V., Magnier S. A., Daudeville L., Mariotti C., Davenne L., « Numerical Study of Compressive Behaviour of Concrete at High Strain Rates », Journal of engineering mechanics, vol. 125, n 10, p. 1154-1163, 1999.

Eurocode, Eurocode 2 - Calcul des structures en béton - Part 1-1:Règles générales et règles pour les batiments, Normes Européennes, 1998.

Frangin E., Marin P., Daudeville L., « Coupled finite/discrete element method to analyze localized impact on reinforced concrete structure », in G. Meschke, R. de Borst, H. Mang, N. Bicanic (eds), Computational Modelling of Concrete Structures, EURO-C, 2006. 
Hentz S., Modélisation d'une structure en béton armé soumise à un choc par la méthode des éléménts discrets, $\mathrm{PhD}$ thesis, Université Joseph Fourier, October, 2003.

Hentz S., Daudeville L., Donzé F., « Discrete Element Modelling of Concrete Submitted To Dynamic Loading at High Strain Rates », Computers and Structures, vol. 82, p. 2509-2524, 2004a.

Hentz S., Daudeville L., Donze F. V., « Identification and Validation of a Discrete Element Model for Concrete », Journal of engineering mechanics, vol. 130, p. 709-719, June, 2004b.

Hentz S., Donze F. V., Daudeville L., « Discrete element modelling of a reinforced concrete structure submitted to a rock impact », Italian Geotechnical Journal, vol. 4, p. 83-94, 2005.

Li S., Liu W. K., Meshfree Particle Methods, Springer, 2004.

Oñate E., Rojek J., « Combination of discrete element and finite element methods for dynamics analysis of geomechanics problems », Comp. Meth. Appl. Mech. Eng., vol. 193, p. $3087-$ 3128, 2004.

Rudd R., Broughton J., « Coarse-grained molecular dynamics and the atomistic limit of finite elements », Physical review B, vol. 58, n 10, p. 5893-5896, September, 1998.

Xiao S., Belytschko T., « A bridging domain method for coupling continua with molecular dynamics », Computer methods in apllied mechanics and engineering, vol. 193, p. 16451669, 2004.

Zineddin M., Krauthammer T., « Dynamic response and behavior of reinforced concrete slabs under impact loading », Int. J. of impact engineering, 2007. 
